\title{
Compression, Tension and Shear Testing of Fibrous Composite with the Split Hopkinson Bar Technique
}

\author{
Amos Gilat ${ }^{*}$ and Jeremy D. Seidt \\ The Ohio State University, Department of Mechanical and Aerospace Engineering, Columbus, OH, 43210, USA
}

\begin{abstract}
The Split Hopkinson Bar (SHB) technique is used for high strain rate testing of T800/F3900 composite in compression, tension and shear. Digital Image Correlation (DIC) is used for measuring the full-field deformation on the surface of the specimen by using Shimadzu HPV-X2 high-speed video camera. Compression tests have been done on specimens machined from a unidirectional laminate in the $0^{\circ}$ and $90^{\circ}$ directions. Tensile tests were done in the $90^{\circ}$ direction. Shear tests were done by using a notched specimen in a compression SHB apparatus. To study the effect of strain rate, quasi-static testing was also done using DIC and specimens with the same geometry as in the SHB tests. The results show that the DIC technique provides accurate strain measurements even at strains that are smaller than $1 \%$. No strain rate effect is observed in compression in the $0^{\circ}$ direction and significant strain rate effects are observed in compression and tension in the $90^{\circ}$ direction, and in shear.
\end{abstract}

\section{Introduction}

The Split Hopkinson Bar (SHB) technique is frequently used for testing metals at high strain rates in the range of 500 to $5,000 \mathrm{~s}-1$. In this technique a short specimen is placed (or attached) between two metal bars (incident and transmitter). The specimen is loaded by a wave that is generated in the incident bar. Once the specimen is loaded, most of the wave is reflected back to the incident bar and a portion of the wave passes through the specimen to the transmitter bar. The SHB technique was introduced with compression loading by Kolsky [1] and is also used in tension and torsion [2-3]. Traditionally, the stress and strain in the specimen have been determined from the waves in the bars which remain elastic throughout the test, assuming that the stress and deformation in the specimen are uniform. The deformation determined from analysing the waves is reasonably accurate at large strains as the duration of the test is much longer than the time it takes for the specimen to reach equilibrium. At small strains the strain determined from the waves might not be accurate. Compression tests are the easiest to conduct with the SHB technique since the specimen is just placed between the two bars. In tension and shear the specimen has to be mechanically attached to the bars. This effects the state of stress and deformation in the specimen and also has an effect on the transmission of the waves from the specimen's ends to the bars.

Testing of fibrous composites with the SHB technique requires special attention due to the nonhomogeneous structure of the material, the requirement of specimens of small size, and the brittleness of the material. Small fibrous composite specimens are difficult to machine and attach to the testing apparatus. Brittleness of fibrous composites is another issue that has to be addressed when testing with the SHB technique. In many cases a composite specimen fractures at strain of less than $5 \%$ and sometimes at strains of the order of $1 \%$ or less. Determining such small strains in SHB experiments from the waves in the bars might not be accurate due to the effect wave dispersion and the effect of the specimen boundary and attachment method on the waves.

In recent years Digital Image Correlation (DIC) technique has been introduced for accurate full-field measurement of strains directly on the surface of the specimen [4]. The technique was used in the SHB technique using high speed cameras for testing a ductile material (copper) in tension and compression by Gilat et.al. [5]. The results show that in compression the strains determined from the waves agree with the DIC measured strains, but in tension there are large discrepancies.

In most of the data published from SHB tests with fibrous composites the deformation was determined from the elastic waves in the bars. In some investigations the strain was also measured with foil strain gauges that were placed on the surface of the specimen. Compression SHB tests have been presented in [6-8], and tensile tests in [9-10]. Recently, with the availability of high-speed cameras, full-field deformations were measured using the DIC technique in compression SHB tests [11] and using the DSP (digital speckle photography) method in tensile SHB tests [12].

In the present paper specimens made of T800/F3900 composite are tested in compression, tension, and shear at high and low strain rates. The high strain rate tests are

Corresponding author: gilat.1@,osu.edu 
done using the SHB technique and the low rate tests are don using a hydraulic frame. In all the tests full-field deformation is measured on the surface of the specimen using the DIC technique.

\section{Experimental setup}

\subsection{Split Hopkinson (Kolsky) Bar}

The compression and tensile SHB experiments are shown schematically in Figure 1. The compression setup consists of $12.7 \mathrm{~mm}$ diameter titanium $6 \mathrm{Al}-4 \mathrm{~V}$ bars and the tensile apparatus consists of $12.7 \mathrm{~mm}$ diameter 7075 aluminium bars. Loading in compression is generated by impact of a bar and tensile loading is generated by the release of a stored tensile force in a section of the input bar [13]. The waves in the compression apparatus are measured at the midpoint on the incidents and transmitted bars. In the tensile test the waves are measured at two locations on the incident bar and one location on the transmitter bar. The surface of the specimens is painted with white and black speckle pattern and a Shimadzu HPV-X2 high-speed video camera (frame rate of up to $10,000,000 \mathrm{fps}$ and $400 \times 250$ pixel resolution) is used to record images of the deforming specimen. A commercial 2D DIC software (VIC-2D by Correlated Solutions) is used for determining the strains. The force in the specimen is determined from the wave recorded in the transmitter bar.

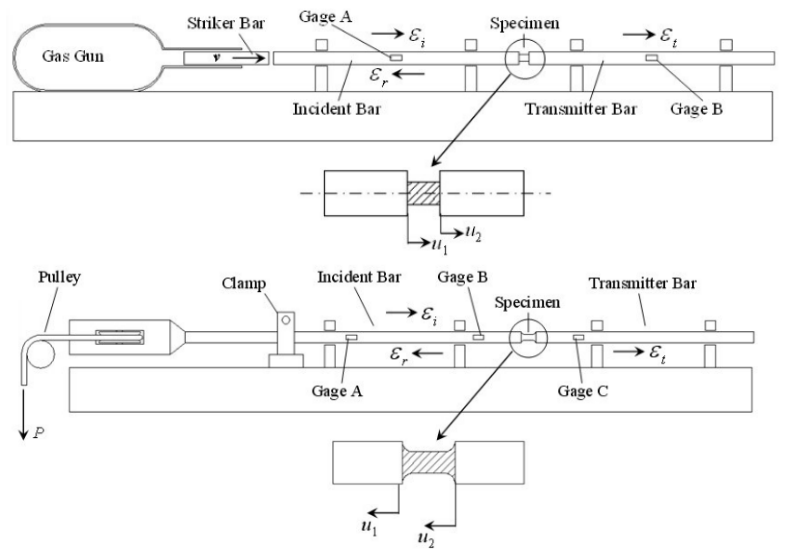

Fig. 1. Schematics of the compression (top) and the tensile (bottom) SHB apparatus.

\subsection{Specimens}

The specimens are machined from a $3.18 \mathrm{~mm}$ thick T800/F3900 unidirectional plate. For the compression tests the specimen is a short small rectangular prism, Figure 2. The prisms are machined in the $0^{\circ}$ or $90^{\circ}$ directions. To prevent transverse motion of the specimens during the compression tests, titanium platens with a shallow $0.5 \mathrm{~mm}$ deep rectangular notch, Figure 2, are used. The specimen ends are inserted into the notches and the assembly is then placed between the input and transmitter bars or in the hydraulic frame.

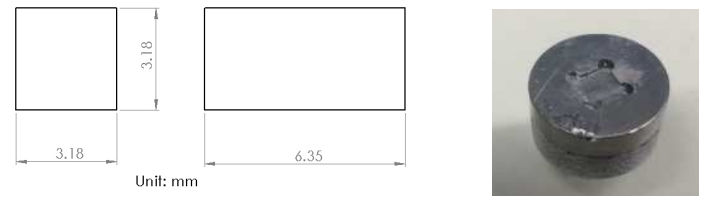

Fig. 2. Compression specimen geometry (left). Titanium platen (right).

The specimens for the tensile tests are flat $3.18 \mathrm{~mm}$ thick with a dog-bone geometry according to ASTM 638-02a, Figure 3. Tensile tests were done in the $90^{\circ}$ direction. The gauge section is in the middle and the wide tabs are glued into notched aluminium adaptors. In the SHB tests the specimen assembly is glued between the incident and transmitter bars. In the low rate tests the assembly is attached with double universal joints to the hydraulic frame.

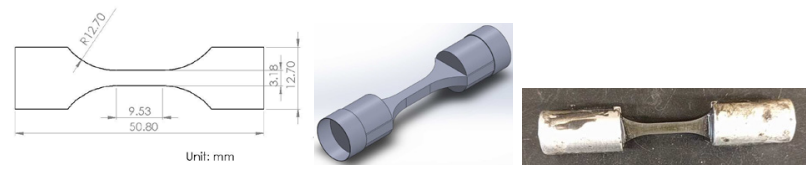

Fig. 3. Tensile specimen geometry (left), specimen with adaptors (middle), photo (right).

The shear test follows a testing configuration suggested by Yokoyama and Nakai [14]. A notched specimen, shown in Figure 4, is machined from the plate such that the fibers are in the direction along the width. The specimen is placed in a compression SHB apparatus. The shear force in the gauge section is determined from the force measured in the transmitter bar and the strain is measured with DIC.
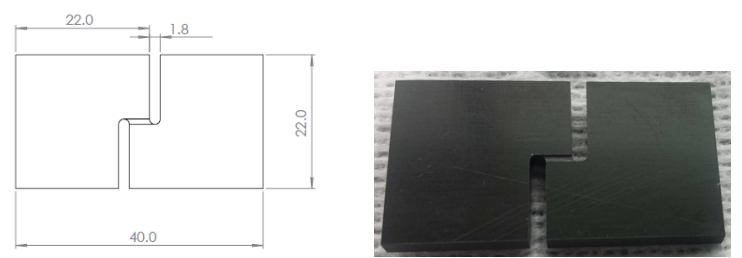

Fig. 4. Shear specimen.

\subsection{Low strain rate tests}

Low strain rate tests were done using a hydraulic MTS testing machine. In order to eliminate a possible effect of the specimen geometry on the data, specimens with the same geometry as in the SHB tests were used.

\section{Results}

Stress strain curves from high and low strain rates $0^{\circ}$ compression tests are shown in Figure 5. Strain versus time plots from the SHB tests are shown in Figure 6. The strain in the figures is obtained by averaging the strain on one surface of the specimen. An image from the DIC measurements of one surface of the specimen is shown in Figure 7. The image shows one frame in the middle of a test. The image shows that the axial strain is essentially uniform, except at the very ends of the specimen where 
the strains are larger (purple color). Comparison of the stress strain curves from the low and high strain rate tests shows no strain rate sensitivity. The strain versus time plot in Figure 6 shows an increasing strain rate (slope) in the first $7 \mu \mathrm{s}$. This is followed by an approximately constant strain rate that is estimated by the dashed line to be $730 \mathrm{~s}^{-1}$.

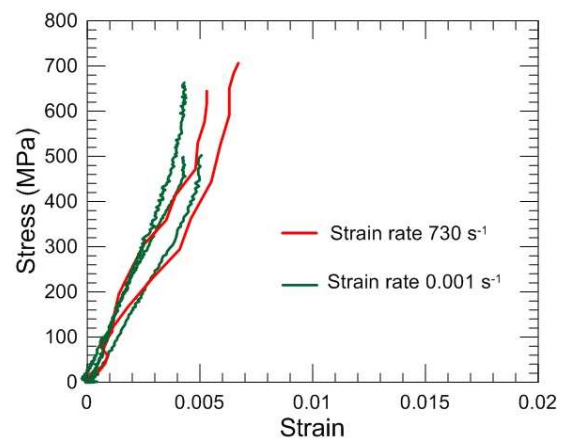

Fig. 5. $0^{\circ}$ direction compression stress strain curves.

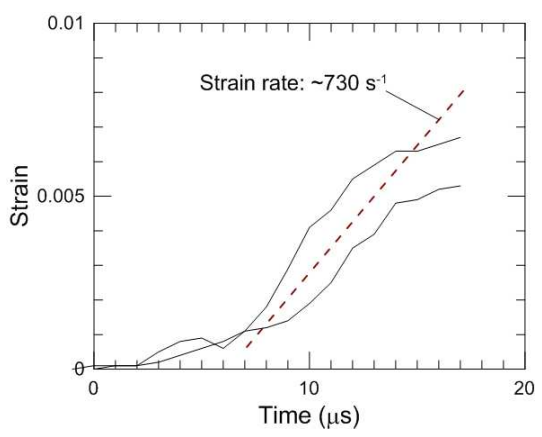

Fig. 6. SHB $0^{\circ}$ direction compression strain vs time.

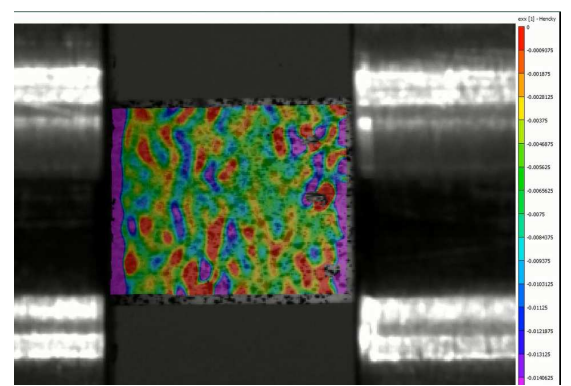

Fig. 7. DIC image from $\mathrm{SHB} 0^{\circ}$ direction compression test.

Stress strain curves from high and low strain rates $90^{\circ}$ compression tests are shown in Figure 8. Strain versus time plots from the SHB tests are shown in Figure 9. In this test the specimens were positioned such that the DIC measurements were done on two surfaces of the specimen. The strain in the figures is obtained by averaging the strain on one surface of the specimen. A DIC image in the middle of the test as the specimen is loaded is shown in Figure 10. It shows that the axial strain is essentially uniform on each of the surfaces. However, there seems to be a small difference in the strain between the two surfaces. This might be due to a small misalignment between the specimen end surfaces and the SHB bars. At the very ends of the specimen the strains are larger (purple colour). Comparison on the data from the low and high strain rate tests shows significant strain rate effect. The maximum stress at the high strain rate test is almost $150 \mathrm{MPa}$ higher (70\%) than in the low strain rate test and maximum strain is smaller by about $1 \%$.

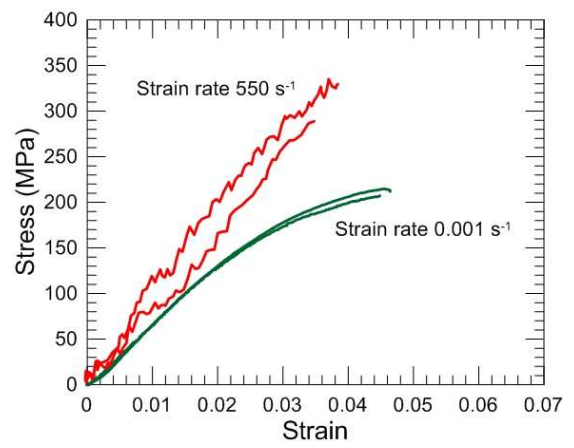

Fig. 8. $90^{\circ}$ direction compression stress strain curves.

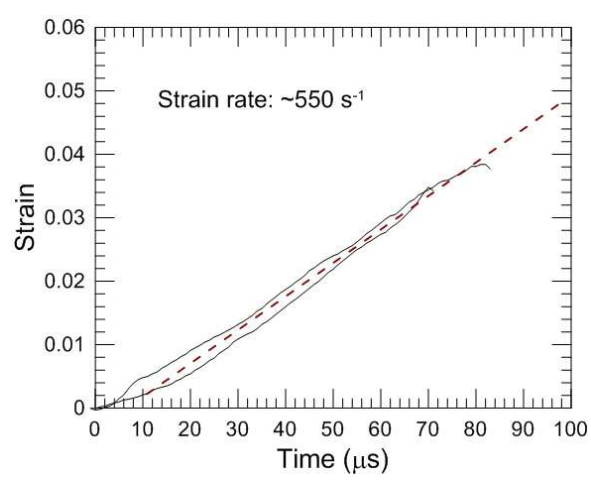

Fig. 9. SHB $90^{\circ}$ direction compression strain vs time.

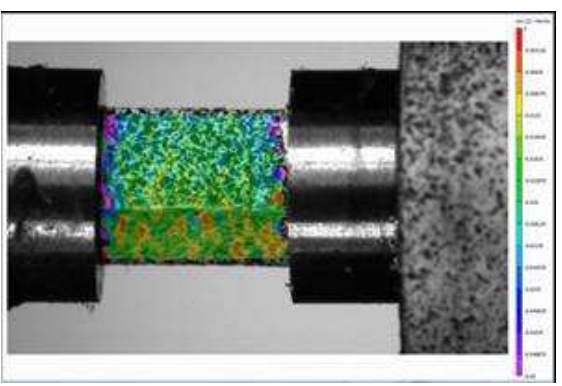

Fig. 10. DIC image from SHB $90^{\circ}$ direction compression test.

Stress strain curves from $90^{\circ}$ tensile tests at high and low strain rates are shown in Figure 11. Strain versus time plots from the SHB tests are shown in Figure 12. The strain in the figures is obtained by averaging the strain on one surface of the specimen. An image from the DIC measurements on the surface of the specimen is shown in Figure 13. The image shows the axial strain at one instant during the loading. It shows uniform strain in the gauge section. As can be seen, the strain at the rounded ends is very small. Comparison of the stress strain curves from the low and high strain rate tests shows significant strain rate effect. At low strain rate the curve is essentially straight with constant slope (modulus). The high strain rate curve appears to have two parts with nearly straight lines. The first part corresponds to the initial modulus which is significantly higher than the modulus at low strain rate. This is followed by a transition to a curve that is nearly parallel 
to the low strain rate curve. The maximum stress at the high and low strain rate tests is about the same, but the maximum strain at the high strain rate tests is about half the value in the low strain rate tests. From Figure 12, the strain rate in the SHB tests is estimated to be $360 \mathrm{~s}^{-1}$.

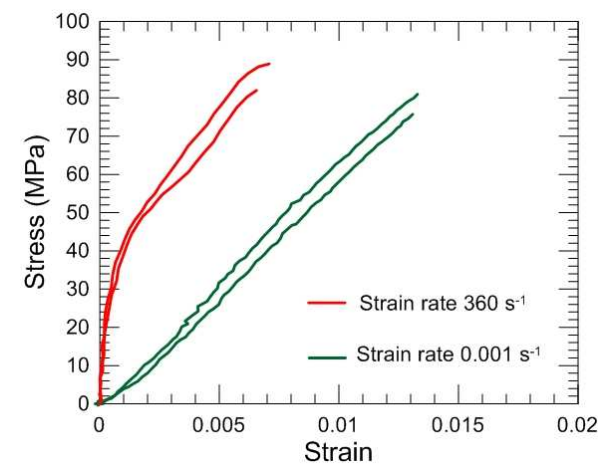

Fig. 11. $90^{\circ}$ direction tensile stress strain curves.

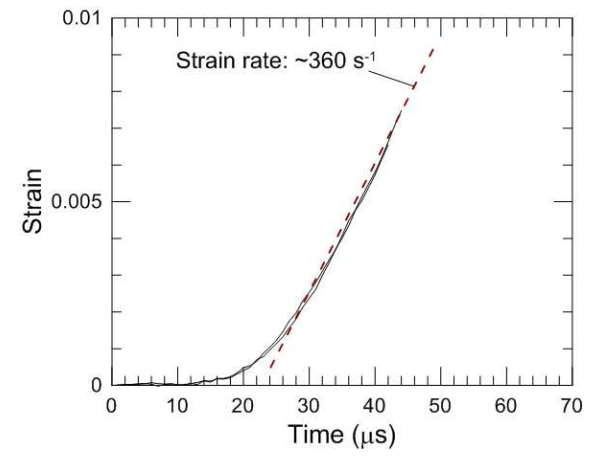

Fig. 12. SHB $90^{\circ}$ direction tensile strain vs time.

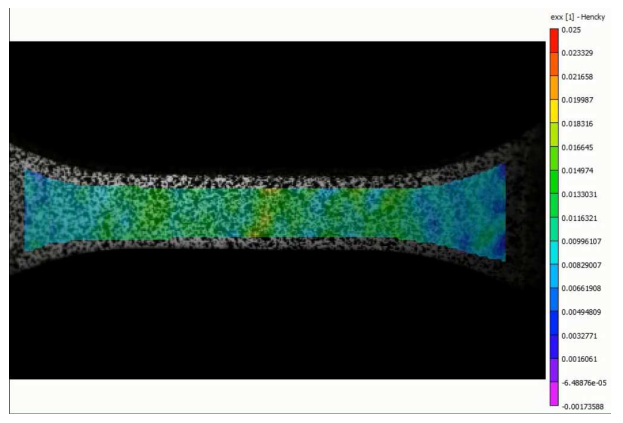

Fig. 13. DIC image from SHB $90^{\circ}$ direction tensile test.

The results from the shear tests are preliminary and additional testing will be done in the future to validate the results. Stress strain curves from the shear tests at high and low strain rates are shown in Figure 14. Strain versus time plot from the SHB tests are shown in Figure 15. The strain $\left(\varepsilon_{\mathrm{xy}}\right)$ in the figures is obtained by averaging the strain on the surface of the notch. An image from the DIC measurements on the surface of the specimen is shown in Figure 16. The image shows the shear strain at one instant during the loading. It shows nearly uniform strain in the gauge section.

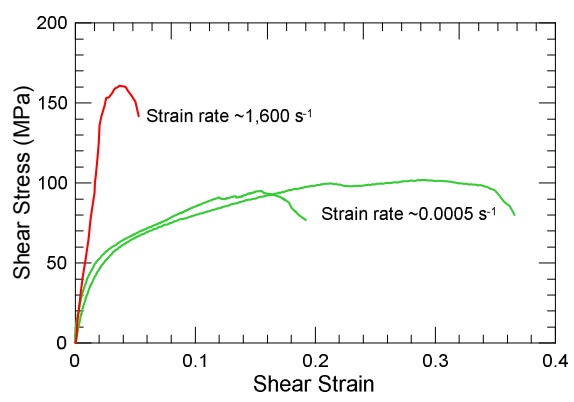

Fig. 14. Shear stress strain curves.

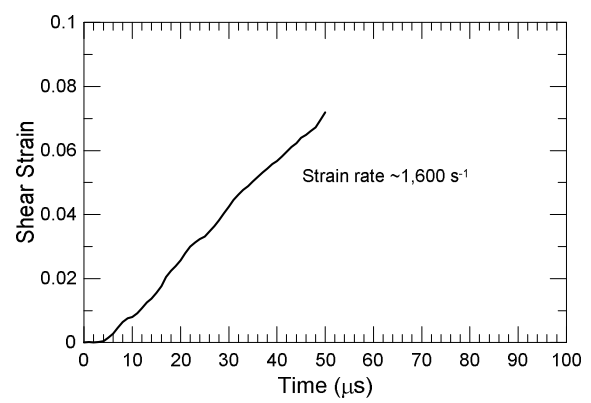

Fig. 15. SHB shear strain vs time.

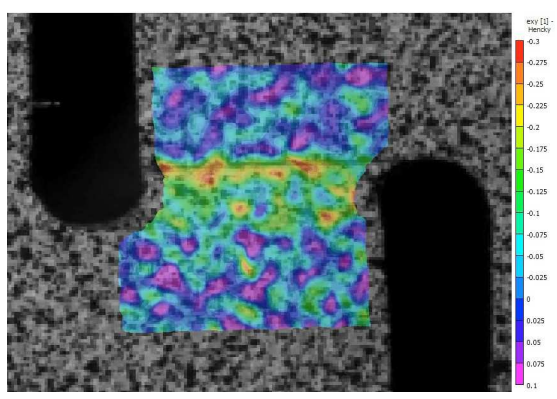

Fig. 16. DIC image from SHB shear test.

\section{Conclusions}

By using the DIC technique with very high speed cameras the split Hopkinson bar technique can be used for studying the response of fibrous composites at high strain rates. Results from testing uniaxial T800/F3900 composite plate shows no strain rate effect in compression the $0^{\circ}$ direction but significant strain rate effects in in compression and tension in the $90^{\circ}$ direction, and in shear.

The research presented was supported by the U.S.A. Federal Aviation Administration, Grants No. 11-G-003 and 16-G007. The authors are grateful to Mr. Bill Emmerling, Dr. Chip Queitzsch, and Mr. Daniel Cordasco of the FAA. The paper is based on research done by Mr. Peiyu Yang (MSc research) and Mr. Yogesh Deshpande (MSc candidate).

\section{References}

1. H. Kolsky, Proceedings of the Physical Society. Section B, 62, 676 (1949)

2. G.T. Gray, ASM Handbook, Mechanical Testing and Evaluation, ASM International, Materials Park, 8, 462 (2000) 
3. A. Gilat, ASM Handbook, Mechanical Testing and Evaluation, ASM International, Materials Park, 8, 505 (2000)

4. M.A. Sutton, J.J. Orteu, and H.W. Schreier, Image correlation for shape, motion and deformation measurements: basic concepts, theory and applications (Springer, New York, 2009)

5. A. Gilat, T.E. Schmidt, and A.L. Walker, Exp Mech 49, 291 (2009)

6. L. Ninan, J. Tsai, and C.T. Sun, Int J Imp Eng 25, 291 (2001)

7. I.M. Daniel, J.M. Cho, B.T. Werner, and J.S. Fenner, AIAA J., 49, 8 (2011)

8. H. Koerber, and P.P. Camanho, Comp: Part A, 42, 462 (2011)

9. J. Harding, and L.M. Welsh, A tensile testing technique for fibre-reinforced composites at impact rates of strain, Journal of Materials Science, 18, pp. 1810-1826 (1983)

10. H. Eskandari, and J.A. Nemes, J. Comp Mat 34, 260 (2000)

11. H. Koerber, J. Xavier, and P.P. Camanho, Mech Mat, 42, 1004 (2010)

12. R. Gerlach, C.R. Siviour, J. Wiegand, and N. Petrinic, Mech Adv Mat and Str, 20:7, 505 (2013)

13. G.H. Staab, and A. Gilat, A, Exp Mech, 31:3, 232 (1991)

14. T. Yokoyama, and K. Nakai, SEM Con Proc (2006) 
